\title{
Formulation and Evaluation of Floating Microcapsules of Cefpodoxime Proxetil
}

\author{
Monica RP Rao*, Aafaque Yunusi, Swapnil Shelar
}

Department of Pharmaceutics, AISSMS College of Pharmacy, Kennedy Road, Near R.T.O. Pune-411001, Maharashtra, India

\begin{abstract}
Introduction: Floating drug delivery system is widely used technique to obtain higher bioavailability for drugs which have an absorption window in the stomach. Floating microcapsule is one approach to improve gastroretention for oral sustained release dosage forms. Cefpodoxime Proxetil has a biological half life of $2.5 \mathrm{~h}$ and is degraded at higher $\mathrm{pH}$. Objective: Aim of this study was to develop floating microcapsules of Cefpodoxime Proxetil by solvent evaporation technique using Eudragit S100 as a release retarding material. Experimental: A 32 full factorial design was employed to investigate the relationship between concentration of Eudragit and stirring speed on percent release at $12 \mathrm{~h}$, percent entrapment efficiency and particle size. The microcapsules were also characterized for drug content, percentage yield, floatability and surface morphology. Results: The particle size of all batches was found to be in the range of 150-290 $\mu \mathrm{m}$. Increase in concentration of Eudragit S100 was found to increase the entrapment efficiency and mean particle size of the microcapsules. The percent release was found to be higher for microcapsules prepared at higher stirring speed which could be due to lower particle size of microcapsules and hence reduced diffusional path length. All batches of microcapsules remained buoyant for $12 \mathrm{~h}$. It was observed that increasing the polymer concentration caused a decrease in the rate of drug release. The optimum formulation was subjected to pharmacokinetic studies. In vivo studies revealed a 1.6 fold increase in relative bioavailability of Cefpodoxime Proxetil as microcapsules.
\end{abstract}

Key words: Cefpodoxime Proxetil, Floating microcapsule, Solvent evaporation technique, Eudragit S100, Optimization.

\section{INTRODUCTION}

An increasing number of delayed-release and extended-release technologies are available for improving drug therapy via the oral route. These systems are designed to release all or part of their drug load within the lower regions of the gastrointestinal tract, where absorption is typically less efficient. ${ }^{1}$ The Gastro-Retentive Drug Delivery System (GRDDS) is of special interest to improve the bioavailability of drugs that are poorly soluble or unstable at higher $\mathrm{pH}$ of the intestinal or colonic environment. ${ }^{2} \mathrm{~A}$ rational approach to enhance bioavailability and improve pharmacokinetic and pharmacodynamic profile is to retain the drug above its absorption region in GIT, i.e. in the stomach and ensure release of drug in controlled manner for prolonged period of time. ${ }^{3}$ The variability in gastric emptying time leads to unpredictable bioavailability of orally administered dosage forms. ${ }^{4}$ It has been reported that floating drug delivery system is one of the commercial systems which can be used to obtain higher bioavailability than non floating system. ${ }^{5}$ The various approaches for improved gastroretention include floatation, mucoadhesion, sedimentation, unfoldable, expandable, or swellable systems, super porous hydrogel systems and magnetic systems. Every approach has its own limitations, swelling and expanding systems may show a hazard of permanent retention and bio adhesive systems result in irritated mucous layer due to high-localized drug concentration. Single-unit gastro retentive systems exhibit the all-or-none emptying phenomenon.
Submission Date :20-08-14 Revision Date : :12-09-14 Accepted Date : $: 26-11-14$

DOI: $10.5530 /$ ijper.48.4s.13 Correspondence Address Dr. Monica R.P Rao, Department of Pharmaceutics, AISSMS College of Pharmacy, Kennedy Road, NearR.T.O. Pune-411001, Maharashtra, India.

E-mail:monicarp_6@ hotmail.com

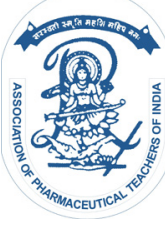

www.ijper.org 
Multiple-unit dosage forms are an alternative since they show a reduction in the inter- and intra-subject variability in drug absorption as well as lower the possibility of dose-dumping characteristics. ${ }^{6-8}$ Floating Drug Delivery Systems (FDDS) have been developed for drugs that act either locally in the stomach or absorbed from it, for those drugs that are either poorly soluble at alkaline $\mathrm{pH}$ or unstable in the intestinal or colonic environment. ${ }^{9-11}$

Cefpodoxime Proxetil (CP) is a prodrug, third generation cephem type broad-spectrum antibiotic administered orally. $\mathrm{CP}$ exhibits $\mathrm{pH}$ dependent solubility behaviour, with highest solubility in acidic $\mathrm{pH} .{ }^{12} \mathrm{CP}$ is used in treatment of acute otitis media respiratory tonsillitis and urinary tract infections, sexually transmitted diseases, skin infections, and pharyngitis. The bioavailability of $\mathrm{CP}$ is only $50 \%$ when given in conventional dosage form as it is stable in acidic $\mathrm{pH}$ and degrades at basic $\mathrm{pH}$. However, the high solubility, chemical and enzymatic stability and absorption profile of $\mathrm{CP}$ in acidic $\mathrm{pH}$ values, points to the potential of a gastroretentive dosage form in altering the absorption profile of $\mathrm{CP}^{13,14}$ In the present study, an attempt was made to develop floating microcapsules of CP using Eudragit S100 as a release retarding material by solvent evaporation technique. The prepared $\mathrm{CP}$ microcapsules were evaluated for drug content, particle size, percentage yield, entrapment efficiency, particle size distribution, surface morphology, In vitro drug release and In vivo studies".

\section{MATERIALS AND METHODS}

\section{Materials}

Cefpodoxime Proxetil (CP) was obtained as a gift sample from Maxim Pharmaceutical Ltd. (Pune, India). Eudragit S100 was supplied by Evonik Industries. Poly Vinyl Alcohol (PVA) supplied by Loba Chemie Pvt Ltd (Mumbai India). All solvents used were of analytical grades and were used as obtained.

\section{Preparation of CP microcapsules}

The microcapsules of CP using Eudragit S100 were prepared by solvent evaporation method. CP and Eudragit $\mathrm{S} 100$ in the ratio $(1: 0.5,1: 1,1: 1.5)$ were dissolved in ethanol:dichloromethane mixture $(1: 1 \mathrm{v} / \mathrm{v}, 10$ $\mathrm{ml}$ ) at room temperature. The drug solution was poured slowly as a thin stream into $200 \mathrm{ml}$ of water containing $1 \% \mathrm{w} / \mathrm{v}$ polyvinyl alcohol. The solution was kept at constant temperature $\left(40^{\circ} \mathrm{C}\right)$ while stirring at $300 \mathrm{rpm}$. The finely dispersed/emulsified droplets of the polymer solution of drug were solidified in the aqueous phase via diffusion of the solvent. After agitating the mixture for $1 \mathrm{~h}$, the microcapsules were filtered, washed several times with water to remove traces of polyvinyl alcohol and dried overnight at $60^{\circ} \mathrm{C}$. Concentration of Eudragit S100 and stirring speed was optimized based on the particle size, percent drug release and percent entrapment efficiency. ${ }^{16,17}$

\section{Experimental Design}

A $3^{2}$ full factorial design was selected for this experiment consisting of 9 design points (A1-A9). The independent variables were stirring speed (X1) and concentration of Eudragit S100 (X2) each at three levels. The dependent variables investigated were the percent drug release after $12 \mathrm{~h}$ (Y1), entrapment efficiency (Y2) and particle size (Y3). The stirring speed levels were 300, 500, $700 \mathrm{rpm}$ while the concentration of Eudragit S100 were 0.5, 1, and $1.5 \mathrm{w} / \mathrm{w}$ and the coded values were $-1,0,1$ respectively (Table 1). The experimental data were analyzed statistically according to established factorial design using Design Expert Software V 9.1 and the main effects and interactions were calculated. The quadratic model was selected. The effect of independent variables on the response parameters were visualised from $3 \mathrm{D}$ response plots. Optimization using desirability approach was employed to locate the optimal settings of the formulation variables to obtain desired response.

\section{Characterization of microcapsules}

\section{Particle size analysis}

The particle size was measured by optical microscopy using Edmundsons equation. ${ }^{15} \mathrm{~A}$ drop of aqueous suspension of microcapsules was mounted on a slide and observed under microscope under 10X magnification. The ocular micrometer was first calibrated with stage micrometer. The mean particle size was calculated by measuring 100 particles with the help of a calibrated ocular micrometer.

$$
\text { Mean particle size. }=\frac{\sum \mathrm{nd}}{\sum \mathrm{n}}
$$

Where $\mathrm{n}$ is no. of particle, $\mathrm{D}$ is diameter

\section{Determination of percent entrapment efficiency and percentage yield}

$\mathrm{CP}$ microcapsules equivalent to $200 \mathrm{mg}$ of $\mathrm{CP}$ was accurately weighed, extracted with $100 \mathrm{ml}$ ethanol by vortexing and allowed to stand for $24 \mathrm{~h}$. The dispersion was filtered through $0.45 \mu$ membrane filter and drug content was determined by UV spectrophotometer (Jasco-V530 and V550) at a wavelength of $263 \mathrm{~nm}$. The calibration curve for $\mathrm{CP}$ was constructed by plotting absorbance against the drug concentrations in the range of $10-50 \mu \mathrm{g} / \mathrm{mL}$. The percent yield of microcapsules was determined by mass balance principle as follows: ${ }^{18}$ 
Percent Yield $=\frac{\text { Theoretical weight of drug and polymer }}{\text { Yield Weight of microcapsules }}$

\section{Floating ability}

Floating behaviour of the microcapsules was studied in USP type II dissolution test apparatus by spreading the microcapsule $(100 \mathrm{mg}$ ) on $0.1 \mathrm{M} \mathrm{HCl}$ containing $0.02 \%$ tween 80 as a surfactant. The medium was agitated with a paddle rotating at $100 \mathrm{rpm}$ and maintained at $37^{\circ} \mathrm{C}$. After $12 \mathrm{~h}$, both the floating and the settled portions of microcapsule were collected separately. The microcapsules were dried and weighed. Buoyancy percentage was calculated as the ratio of the mass of the microcapsules that remained floating and the total mass of the microcapsules. ${ }^{18}$

\section{Differential scanning calorimetry}

Microcapsules of optimized batch were accurately weighed onto aluminium pans and hermetically sealed with aluminium lids. Thermogram were obtained at a scanning rate of $10^{\circ} \mathrm{C} / \mathrm{min}$ conducted over a temperature range of $30-300^{\circ} \mathrm{C}$ in the environment of liquid nitrogen. DSC thermogram of CP, ES100 and microcapsules was recorded using Differential scanning calorimeter (DSC 823 Mettler Toledo, Japan).

\section{Surface Morphology}

The Surface Morphology of optimized microcapsules was investigated using Scanning Electron Microscopy (SEM) (JSM-6360A, JEOL, Tokyo, Japan) by mounting on stubs using double sided adhesive tapes. The stubs were then coated with platinum to a thickness of about $10 \AA$ under an argon atmosphere using a gold sputter module in a high vacuum evaporator. Afterwards, the stub containing the coated samples then placed in the scanning electron microscope chamber.

\section{In vitro drug release studies}

The release rate of CP from microcapsules was determined using USP dissolution testing apparatus II (Paddle type). The dissolution test was performed using 900 $\mathrm{ml}$ of $0.1 \mathrm{~N} \mathrm{HCl}$, at $37 \pm 0.5^{\circ} \mathrm{C}$ and $100 \mathrm{rpm} .{ }^{20}$ Microcapsules equivalent to $200 \mathrm{mg}$ of $\mathrm{CP}$ were used for the test. Sample solution was withdrawn from the dissolution apparatus, every $1 \mathrm{~h}$ up to $12 \mathrm{~h}$. Samples were replaced with equivalent volume of dissolution medium. The samples were filtered through Whatman filter paper and solutions after appropriate dilution were analyzed at 263 $\mathrm{nm}$ by UV Spectrophotometer (JASCO, V-550, Japan). Drug release kinetic was determined using DD Solver v1 Software.

\section{In-vivo studies}

Pharmacokinetic studies were carried out as per the protocols approved by the Institutional Animal Ethical Committee (Ref.No. AISSMS/IAEC/13-14/01-31). The animals used for In vivo experiments were male albino rats weighing 250-300 g. Three groups of six rats each were made for the each study. Suspensions of pure $\mathrm{CP}$ and optimized batch of microcapsules of CP in the dose of $10 \mathrm{mg} / \mathrm{kg}$ were administered orally to rats. The rats were anesthetized using ether and the blood samples were withdrawn from retro-orbital vein at 0 (predose), 0.5, 1, 2, 4, 6, 8, and $12 \mathrm{~h}$ in tubes containing anti-coagulant, mixed and centrifuged at $3000 \mathrm{rpm}$ for $20 \mathrm{~min}$. The plasma was separated carefully and stored at $2-10^{\circ} \mathrm{C}$ until drug analysis was carried out by validated bioanalytical HPTLC method. ${ }^{21}$

\section{Bioanalytical HPTLC method}

The samples were spotted on Merck TLC aluminium plates, precoated with silica gel $60 \mathrm{~F}_{254}$ using a Camag Linomat V applicator (Camag, Switzerland) with a Camag $100 \mu \mathrm{l}$ sample syringe under a nitrogen atmosphere. The length of the chromatographic run was $9 \mathrm{~cm}$ and the time required for each run was approximately 25 min. The mobile phase was chloroform-toluene-methanol in a ratio of 3:4:3. Linear ascending development was carried out in a twin trough glass chamber $(10 \times 10)$ $\mathrm{cm}$. Densitometric scanning of the developed plates was performed using Camag TLC scanner III, operated with WINCATS software (Version 1.4.2, Camag) in the absorbance mode at $263 \mathrm{~nm}$. Scanning speed was kept at $4 \mathrm{~mm} / \mathrm{s}$.

\section{RESULT AND DISCUSSIONS}

Preliminary studies were carried out to identify the domain of concentrations of Eudragit S100 and stirring speed required to construct the $3^{2}$ factorial design. Based on these studies, 03 levels of polymer concentration and stirring speed were shortlisted. Emulsion solvent evaporation technique was used to prepare the microcapsules. The required quantity of drug and polymer were dissolved in a mixture of DCM and ethanol which formed an emulsion on addition to water containing 1\% PVA as emulsifying agent. Ethanol being miscible with water migrates to the aqueous phase thereby decreasing the solubility of drug and polymer. Concurrently continuous stirring leads to evaporation of DCM which causes formation of microcapsules. The percentage yield of microcapsules varied from 68.14 to $87.1 \%$, where it was found to be increased with increasing concentration of Eudragit S100 (Table 1). 
Table 1: Characterization of microcapsules $(n=3)$

\begin{tabular}{|l|c|c|c|c|}
\hline Batch & Particle size $(\boldsymbol{\mu m})$ & $\begin{array}{c}\text { Percentage } \\
\text { Entrapment Efficiency }\end{array}$ & Percentage Yield (\%) & $\begin{array}{c}\text { Floating Ability After } \\
\text { 12h } \mathbf{( \% )}\end{array}$ \\
\hline A-1 & $209 \pm 2.5$ & $74.32 \pm 1.2$ & $68.14 \pm 2$ & $84.32 \pm 1.4$ \\
\hline A-2 & $161 \pm 0.9$ & $72.39 \pm 0.9$ & $70.12 \pm 1.5$ & $80.63 \pm 2.5$ \\
\hline A-3 & $150 \pm 2.5$ & $69.86 \pm 1.5$ & $74.56 \pm 1.8$ & $74.23 \pm 2.7$ \\
\hline A-4 & $261 \pm 1.5$ & $81.02 \pm 0.7$ & $77.45 \pm 1.5$ & $87.23 \pm 0.9$ \\
\hline A-5 & $187 \pm 1.7$ & $77.44 \pm 0.9$ & $79.34 \pm 2.5$ & $81.51 \pm 1.3$ \\
\hline A-6 & $162 \pm 0.7$ & $76.43 \pm 1.5$ & $80.31 \pm 1.1$ & $79.26 \pm 1.5$ \\
\hline A-7 & $287 \pm 1.6$ & $93.09 \pm 1.7$ & $81.00 \pm 1.7$ & $90.73 \pm 1.1$ \\
\hline A-8 & $211 \pm 1.8$ & $87.43 \pm 0.8$ & $83.98 \pm 2.3$ & $89.34 \pm 1.9$ \\
\hline A-9 & $183 \pm 0.9$ & $81.03 \pm 1.3$ & $87.1 \pm 0.9$ & $84.5 \pm 1.5$ \\
\hline
\end{tabular}

Table 2: Summary of results of regression analysis

\begin{tabular}{|c|c|c|c|c|c|}
\hline \multicolumn{6}{|c|}{ For percent drug release at $12 \mathrm{~h}$} \\
\hline Model & Model F value & p value & $\mathbf{R}^{2}$ & Adeq. Precision & Std. deviation \\
\hline Quadratic & 47.74 & 0.0046 & 0.9876 & 19.240 & 0.98 \\
\hline \multicolumn{6}{|c|}{$Y 1=+91.24+1.79 * X 1-5.89 * X 2+0.12 * X 1 X 2-0.22 * X 1^{2}+0.58 * X 2^{2}$} \\
\hline \multicolumn{6}{|c|}{ For percent entrapment efficiency } \\
\hline Quadratic & 46.06 & 0.0049 & 0.9871 & 19.747 & 1.37 \\
\hline \multicolumn{6}{|c|}{$Y 2=+78.14-3.52 X 1+7.51^{*} X 2-1.90^{*} X 1 X 2+0.23^{*} X 1^{2}+1.38^{*} X 2^{2}$} \\
\hline \multicolumn{6}{|c|}{ For Particle size } \\
\hline Quadratic & 88.74 & 0.0019 & 0.9933 & 27.669 & 6.24 \\
\hline \multicolumn{6}{|c|}{$Y 3=+188.44-43.67^{*} X 1+26.83^{*} X 2-11.25^{*} X 1 X 2+22.33^{*} X 1^{2}-3.17^{*} X 2^{2}$} \\
\hline
\end{tabular}

\section{Experimental Design}

On the basis of preliminary trials in the present study a $3^{2}$ factorial design was employed to study the effect of independent variables, i.e. stirring speed (X1) and concentration of Eudragit S100 (X2) on dependent variables percent drug release at $12 \mathrm{~h}$, percent entrapment efficiency, and particle size. The polynomial equations generated by the software were useful for predicting the nature and magnitude of effect of each variable on the responses.

Analysis of experimental results was carried out by using Design Expert V 9.1 software. The quadratic model was suggested to run the design. F-values, P-value and model $\mathrm{F}$-value for percent drug release at $12 \mathrm{~h}$, percent entrapment efficiency and particle size were obtained from ANOVA. The selection of model and polynomial equations are listed in (Table 2). For all responses, the Model F-value implied that the quadratic model was significant. The probability values $(\mathrm{p} \leq 0.05)$ indicated that all the model terms across all the responses were significant. "Adeq Precision" measures the signal to noise ratio, a ratio greater than 4 is desirable. The obtained ratio of 19.240 for release at $12 \mathrm{~h}$ indicated an adequate signal and hence the proposed model can be used to navigate the design space. For percent entrapment effi-
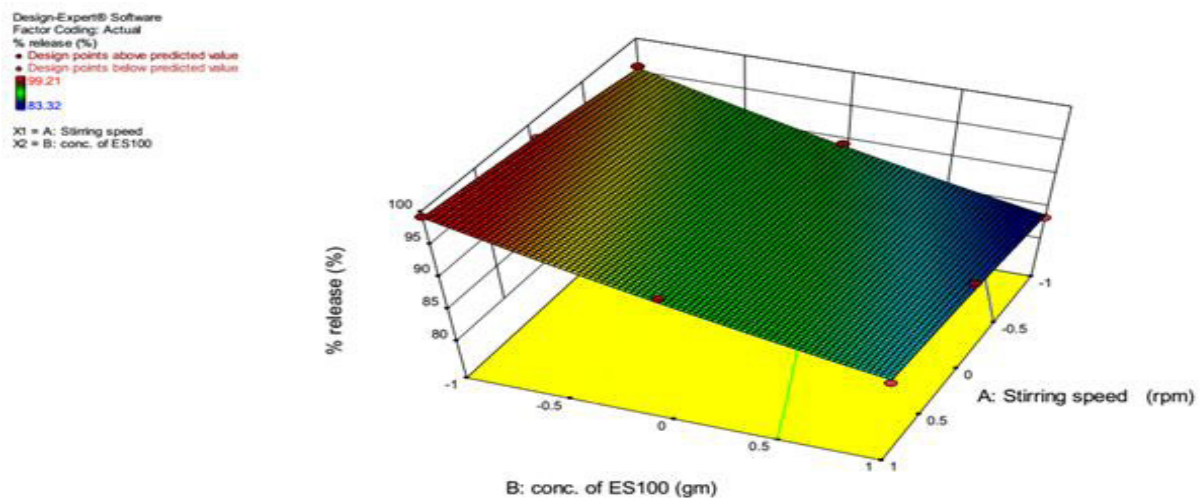

Figure 1: 3-D response surface plots for percent drug release 
ciency and particle size, the ratios were computed as 19.747 and 27.669 , respectively. The polynomial equations for responses Y1, Y2 and Y3 depict the relation between the factors and responses. The coefficients of various terms in the polynomial equations generated by the software give the nature and magnitude of relationship between the variables and the responses.

The 3-D plots (Figure 1) shows that as the concentration of ES100 increases the percent drug release from the microcapsule decreases significantly and as the stirring speed increases the percent drug release get increased from the microcapsule. The equation suggests that factor $\mathrm{X}_{1}$ has positive effect on percent drug release. As level of $\mathrm{X}_{1}$ increases percent drug release also increases. $\mathrm{X}_{2}$ had negative effect on the percent drug release that leads to decrease in the percent drug release as levels of $\mathrm{X}_{2}$ increases.

The drug entrapment efficiency of microcapsules varied from $72.39 \%$ to $93.09 \%$ (Table 1). Results demonstrated that increase in concentration of Eudragit S100 increased the entrapment of the drug. As the $\mathrm{CP}$ has maximum solubility in selected solvent system (i.e.) dichloromethane and ethanol and poorly soluble in aqueous medium, homogeneous solution of drug and polymer obtained for processing and hence drug entrapment was up to its maximum level. The 3-D plots (Figure 2) shows that as the concentration of ES100 increase the percent entrapment efficiency increases significantly and as the stirring speed increases the percent entrapment efficiency decreases. The equation suggests that factor $\mathrm{X}_{1}$ has negative effect on percent entrapment efficiency. As level of $\mathrm{X}_{1}$ decreases percent entrapment efficiency also decreases. $\mathrm{X}_{2}$ had positive effect on the percent entrapment efficiency that leads to increment in the percent entrapment efficiency as levels of $\mathrm{X}_{2}$ increases.

The mean particle size of the microcapsules was found to increase with increasing concentration Eudragit S100 and was in the range $150 \mu \mathrm{m}$ to $290 \mu \mathrm{m}$ (Table 1). This could be attributed to increase viscosity of the medium with increase in Eudragit S100 concentration resulting in enhanced interfacial tension. Shearing efficiency is also diminished at higher viscosities. This leading to formation of larger particles. ${ }^{18}$ The 3-D plots (Figure 3) shows that as the concentration of ES100 increase the particle size increases significantly and as the stirring speed increased the particle size was found to decrease. The equation suggests that factor $\mathrm{X}_{1}$ has negative effect

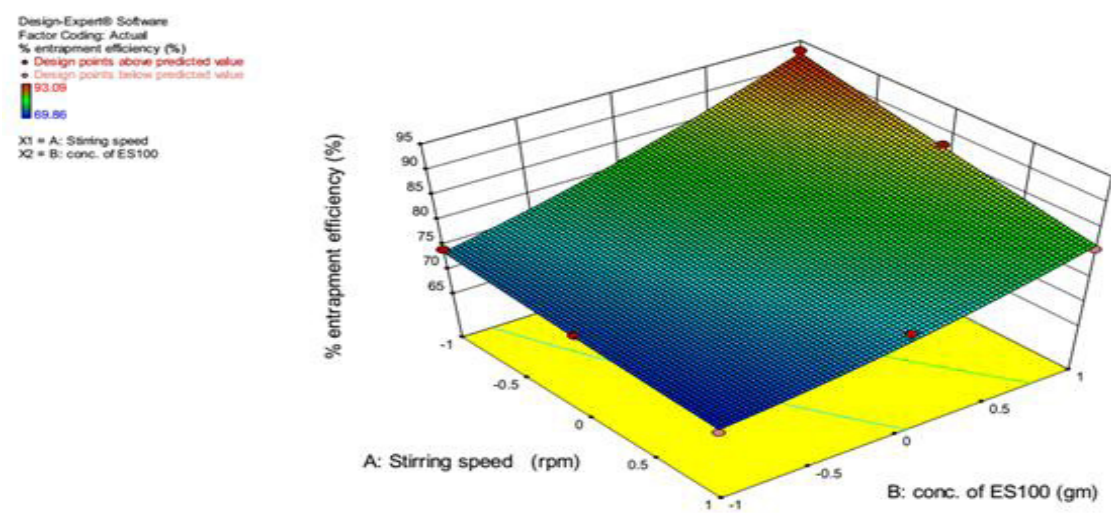

Figure 2: 3-D response surface plots for percent entrapment efficiency
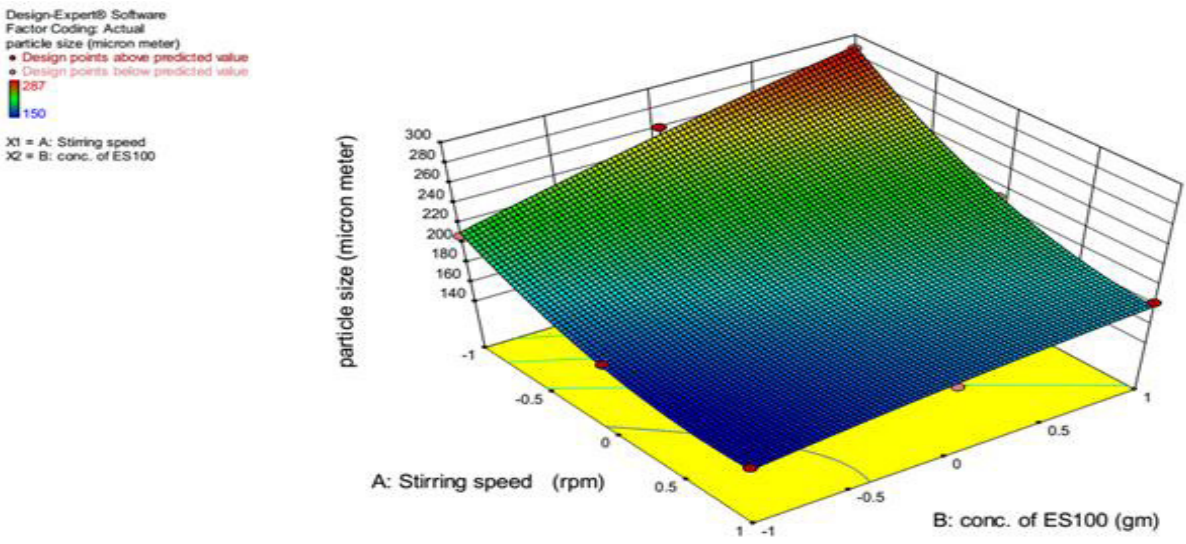

Figure 3: 3-D response surface plots for particle size 


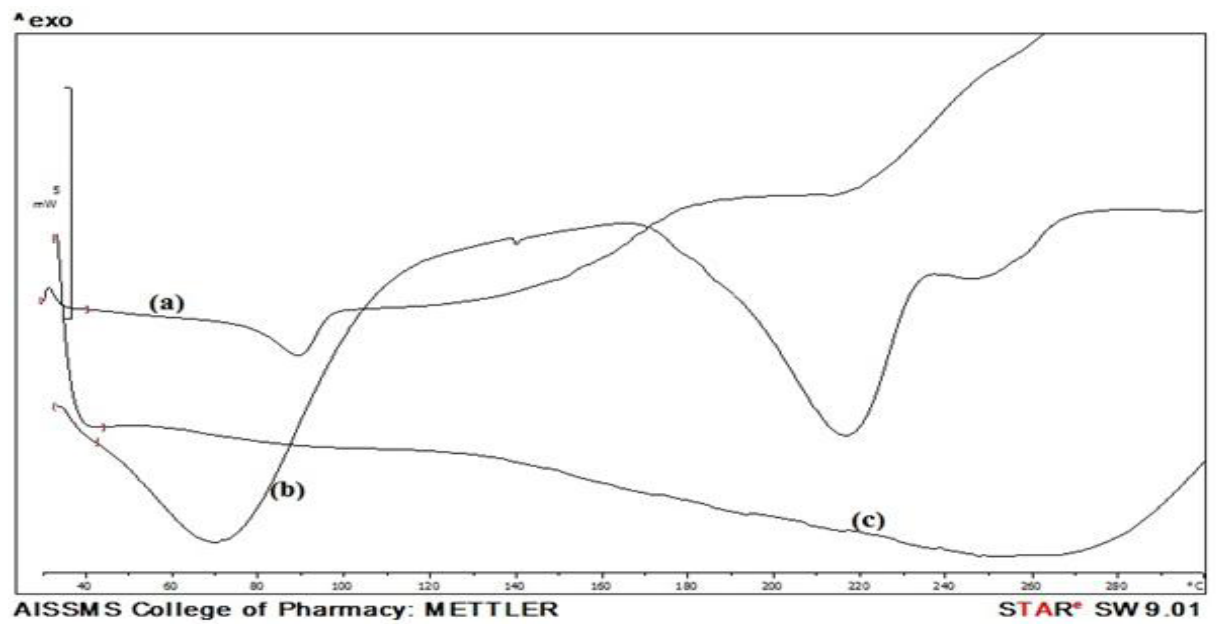

Figure 4: In vitro drug release profiles from CP microcapsules, A-1 to A-9

Table 3: Results of model fitting of batches (A1-A9)

\begin{tabular}{|l|c|c|c|c|c|}
\hline \multirow{2}{*}{ Batch code } & \multicolumn{4}{|c|}{ Regression Analysis } \\
\cline { 2 - 5 } & Zero order & First order & Higuchi & Koremeyer Peppas & Hixon Crowell \\
\hline A-1 & 0.9560 & 0.8484 & 0.7342 & 0.9940 & 0.8847 \\
\hline A-2 & 0.9603 & 0.8479 & 0.7424 & 0.9936 & 0.8860 \\
\hline A-3 & 0.9587 & 0.8456 & 0.7458 & 0.9897 & 0.8848 \\
\hline A-4 & 0.9573 & 0.8570 & 0.7479 & 0.9870 & 0.8922 \\
\hline A-5 & 0.9510 & 0.8586 & 0.7353 & 0.9830 & 0.8840 \\
\hline A-6 & 0.9536 & 0.8464 & 0.7313 & 0.9922 & 0.8840 \\
\hline A-7 & 0.9595 & 0.8737 & 0.7563 & 0.9785 & 0.9059 \\
\hline A-8 & 0.9674 & 0.8800 & 0.7631 & 0.9861 & 0.9126 \\
\hline A-9 & 0.9660 & 0.8804 & 0.7683 & 0.9863 & 0.9126 \\
\hline
\end{tabular}

on particle size. As level of $\mathrm{X}_{1}$ increases particle size decreases. $\mathrm{X}_{2}$ had positive effect on the particle size that leads to increment in the particle size as levels of $\mathrm{X}_{2}$ increases.

\section{Floating ability}

Floating test was carried out to investigate the floatability of the prepared microcapsule. The microcapsules were spread over the surface of simulated gastric fluid and the fraction of microcapsule settled down as a function of time was quantitated. The microcapsules containing Eudragit S100 showed good floating ability range from $74.23 \%$ to $90.73 \%$ (for $12 \mathrm{~h}$ ) as shown in (Table 1) due to insolubility of Eudragit S100 in gastric fluid ( $\mathrm{pH}$ 1.2). Eudragit S100 anionic copolymerization product of methacrylic acid and methyl methacrylate thus affording film coat that is resistant to gastric media and microcapsules are hollow which float on gastric fluid and shows good floatability. ${ }^{19}$

\section{In vitro drug release studies}

In vitro dissolution studies of CP from floating microcapsules were performed in $0.1 \mathrm{~N} \mathrm{HCl}(\mathrm{pH} 1.2)$ for
$12 \mathrm{~h}$ using USP Type II dissolution test apparatus. ${ }^{18} \mathrm{It}$ was found that formulations A1 to A9 showed 97.41 to $67.14 \%$ of release at $12 \mathrm{~h}$ (Figure 4 ). It was observed that as the concentration of Eudragit S100 was increased the percent drug release decreased. The increased Eudragit S100 concentration leads to increased density of polymer matrix in the microcapsules which results in an increased diffusional path length and consequent retardation in drug release. The tortuosity of the path through which the molecules diffuse also increase due to this leading to further decrease in percent release. In case of controlled or sustained release formulations, diffusion, swelling and erosion are the three most important rate controlling mechanisms. Korsmeyer-Peppas was the best fit model which indicates that drug release via a diffusion mechanism because in Korsmeyer-Peppas model drug is dispersed into matrix system and matrix system follows diffusion mechanism of drug release (Table 3).

\section{Optimized batch}

A numerical optimization technique using the desirability approach Design Expert software was employed 


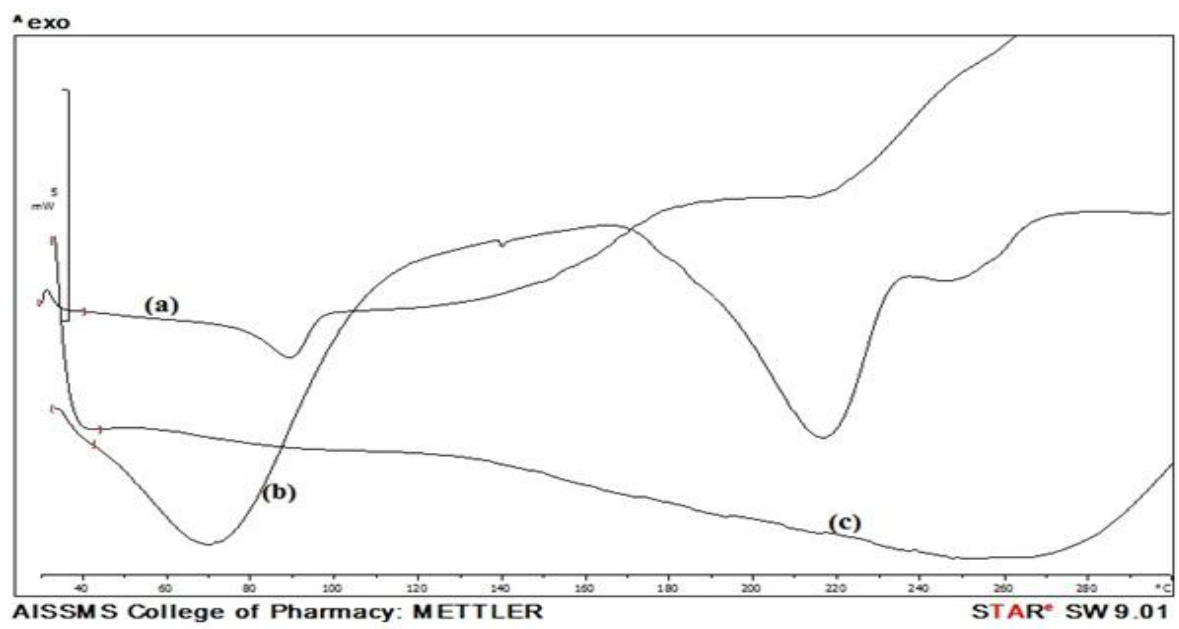

Figure 5: DSC thermogram of (a) CP, (b) Eudragit S100 and (c) Microcapsules

to develop optimum formulation with the desired responses. Constraints were set for maximizing drug release at $12 \mathrm{~h}$, percentage efficiency and particle size to locate the optimum setting of independent variables. Based on the input constraints four optimum formulations were generated by the software. The formulation (S3) which comprised stirring speed (620 rpm) and concentration of Eudragit S100 (1.1 w/w) exhibited the least residual error in the predicted and observed responses for percent drug release at $12 \mathrm{~h}$, percent entrapment efficiency and particle size. Drug release at $12 \mathrm{~h}$, percent entrapment efficiency and particle size from optimized batch was found to be $92.1 \%, 74.4 \%$ and $172 \mu \mathrm{m}$. The optimized batch evaluated further for parameters like differential scanning calorimetry, scanning electron microscopy and In vivo studies.

\section{Differential Scanning Calorimetry}

DSC has been one of the most widely used calorimetric techniques to characterize the physical state of drug in the polymeric matrix. DSC of optimized batch (Figure 5) depicted the DSC thermogram of (a) CP, (b) Eudragit S100 and (c) microcapsule formulation. The DSC thermogram of CP exhibited a single sharp endothermic peak at $89^{\circ} \mathrm{C}$ corresponding to its melting transition temperature and enthalpy value was found to be $10.67 \mathrm{~J} / \mathrm{mol}$. The thermogram of Eudragit S100 exhibited two sharp endothermic peak at $70^{\circ} \mathrm{C}$ and $237^{\circ} \mathrm{C}$ corresponding to its melting transition temperature and enthalpy values were found to be $61.96 \mathrm{~J} / \mathrm{mole}$ and $14.81 \mathrm{~J} /$ mole. The thermogram of Eudragit S100 the based microcapsule showed no such characteristic peak,

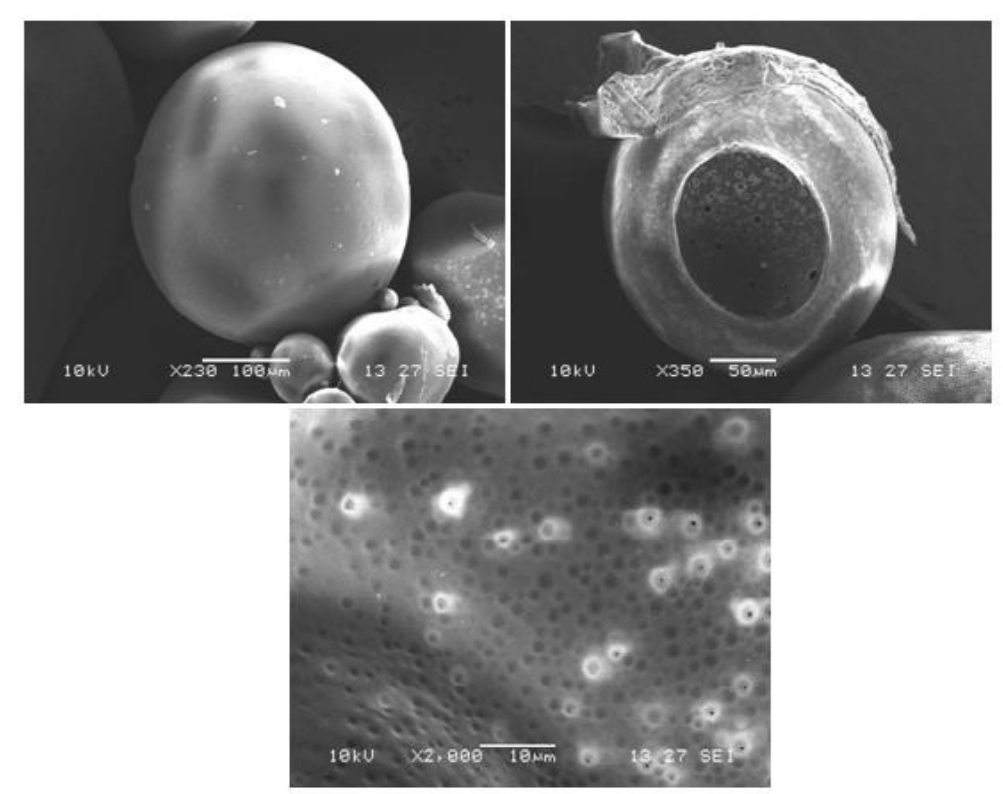

Figure 6: Scanning electron microphotographs of floating microcapsules 


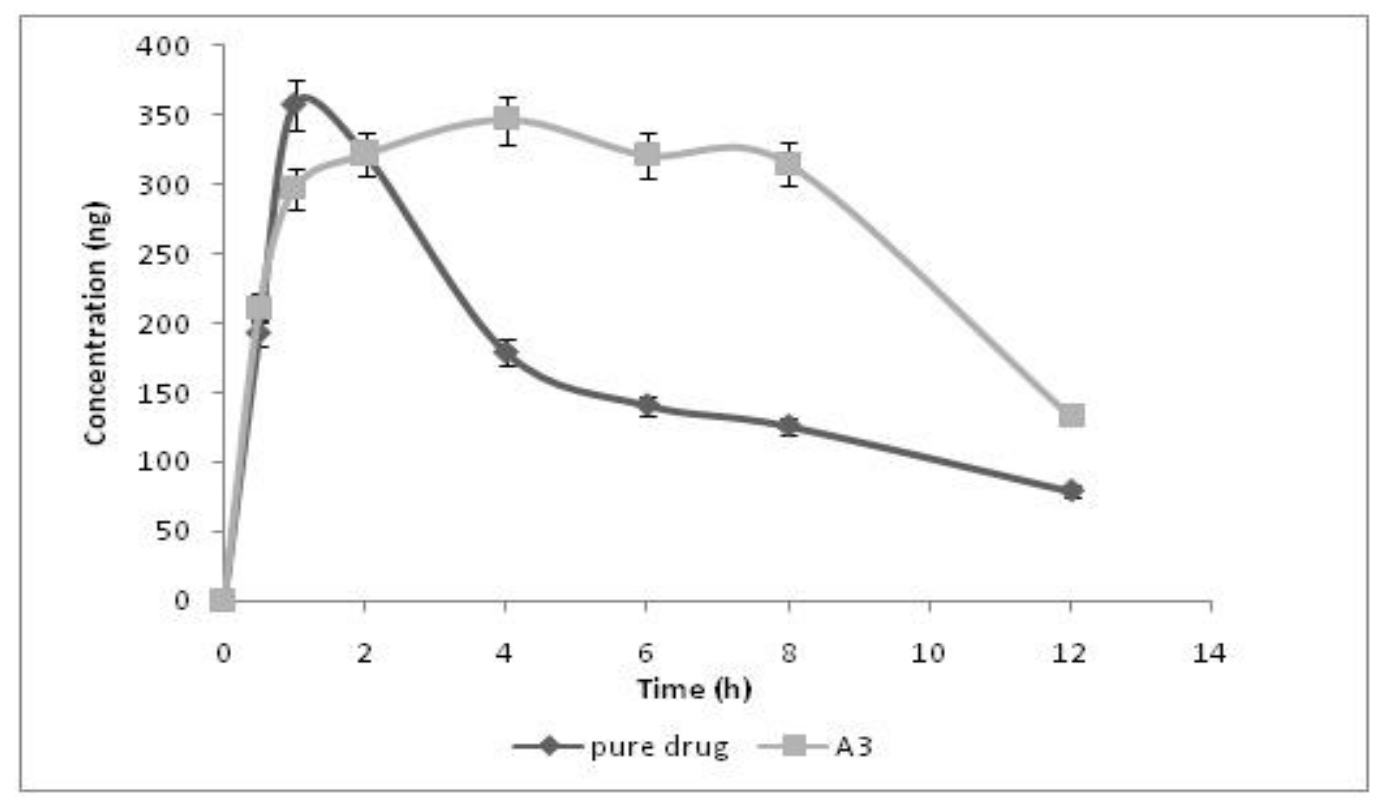

Figure 7: Plasma concentration profile of $\mathrm{CP}$ and $\mathrm{A}-3$ in rats

\begin{tabular}{|l|c|c|c|}
\hline \multicolumn{4}{|c|}{ Table 4: Pharmacokinetic parameters of CP after oral administration of formulation (S-3) and pure drug in } \\
albino rats $(\mathbf{n = 3})$
\end{tabular}

indicating that the drug was uniformly dispersed at the molecular level in microcapsule.

\section{Surface Morphology}

The SEM of optimized batch of microcapsules displayed hollow spherical structure with a smooth surface morphology (Figure 6). The microcapsules showed good floating ability on the surface of the medium, due to hollow structure of microcapsules. The outer surface of the microcapsules was smooth and dense, while the internal surface was porous. It may be caused by the evaporation of solvent entrapped within the shell of microcapsules after forming a smooth and dense skin layer.

\section{In-vivo studies}

The In vivo studies were performed to quantify $\mathrm{CP}$ after oral administration of $\mathrm{CP}$ microcapsule and pure $\mathrm{CP}$. Plasma concentration time curve of pure drug and microcapsule formulation S-3 were compared to find any change in the pharmacokinetic parameters (Figure 7). For pure drug and microcapsules the peak plasma concentration $\left(\mathrm{C}_{\max }\right)$ were found to be $358 \pm 3.8$ (ng/ $\mathrm{ml})$ and $347 \pm 2.1(\mathrm{ng} / \mathrm{ml})$ respectively. The $\mathrm{T}_{\max }$ for pure drug was $1 \mathrm{~h}$ and for CP microcapsules it was found to be $4 \mathrm{~h}$. The higher $\mathrm{T}_{\max }$ of $\mathrm{CP}$ microcapsule suggests slower absorption which could be due to the sustained release of the drug from microcapsule. On the other hand, the peak plasma concentration $\left(\mathrm{C}_{\max }\right)$ of pure drug and CP microcapsule remained nearly unchanged, but The total area under curve i.e. $\mathrm{AUC}_{0 \text { - }}$ ${ }_{12 \mathrm{~h}}$ for pure drug was calculated as $19.66 \pm 0.9 \mathrm{ng} . \mathrm{h} / \mathrm{ml}$ and for CP microcapsules it was found to be $32.48 \pm$ $1.2 \mathrm{ng} . \mathrm{h} / \mathrm{ml}$ (Table 4). This difference could be due to delayed absorption of the drug due to slow release from the microcapsule formulations. The In vivo pharmacokinetic studies showed increase in AUC and thereby increase in bioavailability of CP microcapsule by 1.6 times as compared to pure CP.

\section{CONCLUSION}

In conclusion, the floating microcapsules of $\mathrm{CP}$ prepared with a suitable polymer Eudragit S100, may provide a convenient dosage form for achieving best performance regarding percentage entrapment efficiency and practical yield. The particle size analysis revealed that the particles were of the size range of $150 \mu \mathrm{m}$ to $290 \mu \mathrm{m}$, showed good flow properties the microcapsules were hollow, as revealed by the scanning electron microscopic studies and had low densities, thus exhibiting excellent buoyancies in simulated gastric fluid. In vitro drug release studies showed that release from the microcapsule get successfully retarded for over 
$12 \mathrm{~h}$. The In vivo studies revealed that the relative bioavailability of the drug increased by more than 1.6 times by formulating it into microcapsules.

\section{ACKNOWLEDGEMENTS}

Authors would like to acknowledge support and counselextend by AISSMS college of pharmacy Dr Ashwini $\mathrm{R}$ madulkar.

\section{CONFLICTS OF INTEREST}

Authors report no conflict of interest,financial or otherwise.

\section{REFERENCES}

1. Abdul WB, Fridrun P, Michael JN et al. The use of formulation technology to assess regional gastrointestinal drug absorption in humans. Euro J. Phar Sci. 2004; 21(2): 179-89

2. Rouge N, Buri P, Doelker. Drug absorption sites in the gastrointestinal and dosage forms for site specific delivery. Int J. Phar. 1996; 136(1):117-39.

3. Gattani YS. Floating multiparticulate drug delivery systems: an overview. Int J. Pharm and Bio Sci. 2010; 1(2): 1-14.

4. Sungthongjeen S, Sriamornsak P, Puttipipatkhachorn S. Design and evaluation of floating multi-layer coated tablets based on gas formation. Euro J. Pharm and Biophar. 2008; 69(1): 255-63.

5. Fukuda M, Nicholas A, McGinity JW. Floating hot-melt extruded tablets for gastroretentive controlled drug release system. J. Cont Rel. 2006; 115(2): 121-29.

6. Malakar J, Amit KN. Formulation and statistical optimization of multiple-unit ibuprofen-loaded buoyant system using 23-factorial design. Chem eng res and des. 2012; 90(11):1834-46.

7. Iannuccelli V, Coppi G, Sansone R et al. Air compartment multiple-unit system for prolonged gastric residence. Part II. In vivo evaluation. Int J. Phar. 1998; 174(1): 55-62.
8. Iannuccelli V, Coppi G, Bernabei MR et al. Air compartment multiple-unit system for prolonged gastric residence. Part II. In vivo evaluation. Int J. Phar. 1998; 174(1): 47-54.

9. Talukder R and Fassihi R. Gastroretentive Delivery Systems: Hollow Beads. Drug Dev and Ind Pharm. 2004; 30(4): 405-12.

10. Kharia AA, Hiremath $\mathrm{SN}$, Singhai $\mathrm{AK}$ et al. Design and Optimization of Floating Drug Delivery System of Acyclovir. Ind J. Phar Sci. 2010; 72(5): 599.

11. Shah SH, Patel JK, Patel NV. Stomach Specific Floating Drug Delivery System: A Review. Int J. Pharm Tech Res. 2009; 1(3): 623-33

12. Kakumanu $\mathrm{V}$, Arora $\mathrm{V}$, Bansal AK. Investigation of factors responsible for low oral bioavailability of cefpodoxime proxetil. Int J. Phar. 2006; 317(2):155-60.

13. Amrutkara PP, Chaudhari PD, Patil SB. Design and In vitro evaluation of multiparticulate floating drug delivery system of zolpidem tartarate. Coll and Sur B: Biointer 2012; 89: 182- 87.

14. Sunil KJ, Awasthi AM, Jain NK, Agarwal GP. Calcium silicate based microspheres of repaglinide for gastroretentive floating drug delivery: Preparation and In vitro characterization. J. Cont Rel. 2005;107(2): 300- 9

15. Martin A, Bustamante P, Chun AHC. Physical Pharmacy, BI Waverly Pvt. Ltd, New Delhi. Edition 4. 1995; 557-58.

16. Yasunori S, Kawashima Y, Hirofumi T, et al. In vitro evaluation of floating and drug releasing behaviors of hollow microspheres (microballoons) prepared by the emulsion solvent diffusion method. Euro J. Phar and Biopharm. 2004; 57(2): 235-43.

17. Kawashima $\mathrm{Y}$, Niwa $\mathrm{T}$, Takeuchi $\mathrm{H}$, et al. Preparation of multiple unit hollow microspheres (microballoons) with acrylic resin containing tranilast and their drug release characteristics (In vitro) and floating behavior (in vivo). J. Cont Rel. 1991; 16(3): 279-89.

18. El-Kamel AH, Sokar MS, Al Gamal SS, et al. Preparation and evaluation of ketoprofen floating oral delivery system. Int J. Pharm. 2001; 220(1): 13-21.

19. Choi BY, Park HJ, Hwang SJ et al. Preparation of alginate beads for floating drug delivery system: effects of CO2 gas-forming agents. Int J. Pharm. 2002; 239(1): 81-91.

20. Mastiholimath VS, Dandagi PM, Gadad AP, et al. In vitro and In vivo evaluation of ranitidine hydrochloride ethyl cellulose floating microparticles. J. Microencap. 2008; 25(5): 307-14.

21. Paresh SD, Milind MN. In vitro and in vivo evaluation of sustained release suspensions of ibuprofen. Int J. Pharm. 1991; 73(2): 157-62. 